Rev. Est. de Políticas Públicas, 5(1): diciembre 2018 - junio 2019, 14-32

http://dx.doi.org/10.5354/0719-6296.2019.51103

ISSN edición web: 0719-6296

CCopyright 2019: Universidad de Chile, Santiago (Chile)

\title{
Dilemas de la Planificación Local de la Educación Pública Chilena
}

\author{
Sebastián Donoso, Felipe Ávila, Victoria Gidi y Evaristo Parada \\ Universidad de Talca-Instituto de Investigación y Desarrollo Educacional
}

\section{Resumen}

El texto analiza el instrumento central de planificación local (Municipal) de la educación pública, el Plan Anual de Desarrollo Educativo Municipal (PADEM), que por veinticinco años se ha aplicado, identificándose sus fortalezas y debilidades, y en razón de ello, levantando propuestas para su mejoramiento. El trabajo se sustenta en una revisión documental, del tema y en entrevistas a responsables de encabezar e implementar este proceso en los municipios. Atendiendo a la complejidad del tema en el frágil escenario de la educación publica, se concluye en la necesidad de reemplazar el PADEM por un instrumento que presente sincronía con los procesos educacionales y pertinencia con el contenido de la educación pública y las visiones actuales de gestión en este campo, buscando que su proyección sea aplicable a la nueva institucionalidad subnacional de educación, los Servicios Locales de Educación, que son unidades territoriales de educación pública, mayores y más complejas que las comunas, no debiendo repetirse los errores y debilidades que arrastra este instrumento para las nuevas estructuras en desarrollo.

Palabras clave: Planificación estratégica, instrumentos de planificación, educación local, educación pública, educación chilena.

\section{Dilemmas of the Local Planning of Chilean Public Education}

\begin{abstract}
The text analyzes the central instrument of local planning of public education (Municipal), the Annual Plan for Municipal Educational Development (PADEM), which for twenty-five years has been applied, identifying its strengths and weaknesses, and as a result, raising proposals for its improvement. The work is based on a documentary review of the subject and interviews with those responsible for leading and implementing this process in the municipalities. Considering the complexity of the issue in the fragile scenario of public education, it is concluded that PADEM needs to replace an instrument that is in sync with the educational processes and relevance to the content of public education and current management visions. this field, looking for its projection to be applicable to the new subnational institutionality of education, the Local Education Services, which are territorial units of public education, larger and more complex than the communes, and should not repeat the errors and weaknesses that this instrument carries for new structures in development.
\end{abstract}

Keywords: Strategic planning, planning instruments, local education, public education, Chilean education.

*Dirección de correspondencia [Correspondence address]:

Sebastiàn Donoso, Universidad de Talca

E-mail: donoso.sebastian@gmail.com 


\section{Presentación}

La educación pública chilena desde la reforma de 1980, sustentada en una visión de mercado neoliberal, ha tenido una persistente caída en indicadores claves, a saber, la matrícula escolar pasó de atender al $78 \%$ de los estudiantes -en1980- al 36,5\% el año 2017 (MINEDUC, 2018), acompañada de un transitar complejo en los resultados de aprendizaje en las pruebas nacionales e internacionales, con impactos sustantivos en la gestión y financiamiento del sector que han conformado un cuadro crítico con implicancia relevantes sobre su viabilidad futura (OCDE, 2017).

Entre las transformaciones estructurales impulsadas por la reforma en comento, destaca el traspaso de los establecimientos escolares públicos, personal docente y administrativo desde el aparato central del Estado (Ministerio de Educación) a los municipios, como también el cambio de los principios e instrumentos de financiamiento del sector, pasando de un modelo de financiamiento a la oferta a uno sustentado en la demanda. Medida adoptada súbita y sin previo pilotaje, que involucró en su primer año al $70 \%$ de las escuelas y el saldo restante un par de años después. Adicionalmente en este proceso, los Municipios recibieron escaso apoyo del Gobierno central para asumir las nuevas tareas de gestión, con clara inexperiencia de los Gobiernos locales en el manejo del nuevo sistema de subsidio financiero, todo ello en un marco político diseñado para estimular la privatización del sector (Jofré, 1988; Joiko, 2012).

La reforma señalada entregó a los municipios la gestión de la educación pública, sin dotarla de instrumentos adecuados para asumir y proyectar esa tarea. Una década y media después de iniciado este camino -en 1995-, el Gobierno definió el Plan Anual de Educación Municipal (PADEM) como el instrumento central de planificación de la educación pública local, estableciendo su obligatoriedad como tarea anual que los municipios deben realizar (Waissbluth, 2013; Larrañaga et al., 2009).

A comienzos del presente siglo, los movimientos estudiantiles de 2006 y 2011 instalaron en el debate nacional demandas sustantivas en el plano del fortalecimiento de la educación pública escolar y superior (atender estas problemáticas propusieron políticas que derivaron, entre otras aspectos, en la nueva ley que creó el Sistema de Educación Pública (noviembre 2017), cuya implementación comenzó en forma gradual el 2018. La ley señalada implica que al menos durante la próxima década sino más, funcionará de manera simultánea la actual estructura municipal $\left(\right.$ DAEM $^{1}$ ) junto con la nueva institucionalidad: los Servicios Locales de Educación Pública (SLE), instancia que paulatinamente debiese absorber al sistema municipal ${ }^{2}$.

De esta manera, el actual instrumento de planificación -el PADEM- debiese tener un horizonte de vigencia importante (hasta el año 2030 para las comunas no incorporadas a los SLE), no obstante en forma paralela los SLE tienen que elaborar un Plan Estratégico de Desarrollo (MINEDUC, 2017), con la misma finalidad pero con plazos de aplicación más extensos y otros procedimientos de validación de la información, en razón de lo cual es pertinente y además relevante revisar el proceso de desarrollo del PADEM -desde sus actores locales responsables- para corregir sus limitaciones y obstáculos, y no replicarles en una escala de mayor magnitud como son los SLE, dado que cada uno de éstos agrupará en promedio entre 4 -6 comunas (de 346 sistemas comunales a 70 SLE).

Adicionalmente, el estudio del PADEM, sus limitaciones y obstáculos, es importante dado que sus fundamentos y operatoria provienen de la Gestión Pública convencional, como se verá más adelante, distantes de las características más propias del sector educativo, evidenciando dificultades de diseño y sincronización con el sector educación y sus prácticas de operación, como también de sinergia con otros instrumentos de planificación que se manejan a nivel de la comuna (Plan de Desarrollo Comunal PLADECO), y con los planes de cada establecimiento escolar (Proyecto Educativo Institucional- PEI y Proyecto de Mejoramiento Educativo- PME), dando cuenta de problemáticas que deben enfrentarse en el contexto actual de los sistema de planificación democrática de gestión local, escenario en el que la educación pública chilena está en deuda, por la obsolescencia de los instrumentos actualmente en uso con el contexto social, económico y político en que se sitúa el país (Donoso et al., 2018).

Atendiendo lo señalado, el foco del estudio es la precisión de las dificultades y limitaciones que presenta el PADEM a partir de la opinión de aquellos actores claves que han tenido responsabilidad en su aplicación anual en el plano comunal, revisando el

\footnotetext{
${ }^{1}$ Departamento de Administración de la Educación Municipal.

${ }^{2} \mathrm{Si}$ bien la ley estableció un plazo de 12 años para este proceso (2018 - 2030), dejó abierta la posibilidad que el año 2021, una comisión de experto establezca otro calendario, o bien decida detener el proceso o congelarlo, o finalmente mantenerlo, situación que genera incertidumbre.
} 
proceso de diseño, instalación, seguimiento y evaluación, con la finalidad tanto de contribuir a su reemplazo por otro instrumento que responda mejor a las características de la educación pública, como también para aportar -desde esta experiencia- al nuevo instrumento de planificación que deben utilizar los Servicios Locales de Educación.

\section{Problemas de la Institucionalidad Subnacional de la Educación Pú- blica}

La crisis de la educación pública chilena fue definida por el Consejo Asesor ${ }^{3}$ (2006), sosteniéndose que hay tres ámbitos en que la institucionalidad de la educación pública ha sido inapropiada: la eficiencia, equidad y calidad de los resultados educativossobre los cuales no existe pleno consenso, pero hay claridad en términos que "el esquema institucional ha mostrado limitaciones vacíos e inconsistencias" (Consejo Asesor, 2006: 111).

\begin{abstract}
"El Consejo concuerda que el desempeño del actual sistema de administración municipal de la educación es negativo. Hay acuerdo en un conjunto de condiciones que explican las falencias del sistema municipal, un financiamiento insuficiente para las obligaciones que poseen, disolución de responsabilidades entre el Ministerio y los municipios, escasa transferencia de competencias a las escuelas, relación desigual entre el sector municipal y el particular subvencionado. Algunos consejeros señalan que también influye el tamaño y sus capacidades de gestión entre ellos"

(Consejo Asesor, 2006: 115).
\end{abstract}

Un informe de la OCDE (2004) sobre la situación educativa chilena expresó la inconveniencia de la municipalización por su asociación al financiamiento por subsidio a la demanda, y por la preeminencia de este criterio sobre las razones educacionales a la hora de las decisiones finales, llevando la discusión hacia el sistema de financiamiento de la educación. "Las debilidades administrativas de las regiones y municipalidades y la ambigüedad funcional en la división de responsabilidades a través de los niveles

${ }^{3}$ El Consejo Asesor Presidencial para la Calidad de la Educación, fue la instancia definida entonces por el Gobierno (2006) para solucionar a la crisis planteada por el movimiento estudiantil. Su informe final es una pieza insustituible para comprender el contexto y dimensiones del problema tratado. administrativos perjudican una entrega más efectiva de educación de calidad a los niños socialmente marginados" (OCDE, 2004: 279). Además, el informe señaló las escasas atribuciones de las autoridades intermedias (regionales y provinciales) del Ministerio de Educación ante las autoridades nacionales del Ministerio. "El equipo de revisión también notó una debilidad de las estructuras de nivel medio: (regionales y provinciales), que parecen solo administrar los mandatos educacionales del gobierno central" (OCDE, 2004: 280).

El Ministerio de Educación desconcentró sus funciones en sus unidades territoriales pero con un desbalance de poder entre las atribuciones del nivel central y las delegadas a los niveles subnacionales. Ello implica que el Ministerio define planes nacionales sin interactuar necesariamente con los municipios, que son los operadores de los establecimientos escolares, pero no existe la práctica de construir un diseño de planificación de carácter participativo y comprometido con los entes subnacionales, sino desde las propuestas del Ministerio central (Donoso et al., 2018). Producto de las reformas de los 80 el país registra una situación atípica, a saber, posee uno de los sistemas educativos más descentralizados del mundo, en un contexto político de máxima centralización (Politeia, 2008).

La educación nacional en materia de logros educativos avanzó lenta y sistemáticamente, siendo más relevante en su progresión que el factor dependencia del establecimiento (público o privado) el capital socioeconómico de la familia, confirmando que la sociedad chilena es una de las más desiguales de América latina (PNUD, 2017). Estudios muestran desde los complejos impactos del proceso de municipalización en la segregación y desigualdad social (Valenzuela et al., 2014; Bellei et al., 2013; Bellei, 2014; OCDE, 2017; Treviño et al., 2018), hasta la dificultad de insistir en este camino dada la organización del Gobierno local y el papel preponderante y sin contrapeso del jefe comunal (Alcalde) lo que debilita el desarrollo institucional, lo que se denomina la "alcaldización" de la educación pública (Elacqua et al., 2010; Donoso y Benavides, 2017).

El deterioro incremental de la educación pública reside en grado importante en su institucionalidad y en la asimetría derechos/deberes ante la educación particular. "Estas materias se expresan en tres componentes dominantes del sistema: el modelo de financiamiento (subsidio por alumno y financiamiento compartido), la institucionalidad (municipal, y regional) y el desequilibrio entre los recursos asigna- 
dos y las obligaciones que pesan sobre la educación pública" (García-Huidobro, 2010: 84). La búsqueda de soluciones sin salirse del mercado -que impulsaron los gobiernos democráticos- detonó las contradicciones más relevantes en el sector educación, cuya expresión fue tratar de congeniar medidas correctivas de los instrumentos de mercado (Cox, 2003), con políticas de financiamiento de mercado (OCDE, 2004), experimento sin solución, por cuanto las medidas pro mercado fueron dominantes en el sector, confirmando con ello la prevalencia del paradigma económico neoliberal (Atria, 2014; Bellei, 2015). En este escenario los grupos conservadores han insistido en mantener el sistema vigente mejorando el valor del subsidio y reduciendo algunas trabas burocráticas (Eyzaguirre, 2012).

Entonces se podría concluir que el problema central de la educación chilena que es la baja calidad de la enseñanza, su asociado con la alta segregación socioeconómica y territorial del sistema educacional (y social), se vincula poderosamente con la estructura operativa, organizacional y financiera que no es la adecuada para enfrentar con éxito estas tareas (Valenzuela et al., 2014; Treviño et al., 2018; Núñez y Weinstein, 2010). Si bien sus detonantes principales para la educación pública no son solo económicas, no excluyen esta variable, considerando Adams el sentido de la educación pública reducido en la actualidad al marco de los aprendizajes instrumentales y mucho menos relevado su impacto en los procesos formativos.

En referencia a estos ejes han girado los debates políticos de la educación después del 2011, incorporando dimensiones que otrora no se habían considerado, e igualmente dando pie a incidencias entre la organización del Estado, de la educación y de las demandas ciudadanas locales, con aristas diferentes a los esquemas convencionales de agrupación de los actores políticos, que no se condicen con los cánones más conocidos, generando nuevas situaciones relevantes de consignar (Ruiz, 2016; Garretón, 2016), situando la temática de la gobernabilidad del sector en entredicho, que no se reduce a demandas puntuales si no a temas claves sobre lo hecho por el Estado y lo que puede llegar ha hacer bajo distintas concepciones en juego.

\section{El Plan Anual de Desarrollo Edu- cativo Municipal (PADEM)}

Desde hace más de dos décadas los Departamentos de Administración de la Educación Municipal
(DAEM) emplean como instrumento central de planificación anual de sus operaciones el PADEM, el cual supone impulsar un proceso cuyas etapas implican contextualizar la situación del territorio (comuna) de la educación pública y en función de ello planificar las acciones del sector. Desde 1995 (ley $\mathrm{N}^{\circ} 19.410$ ), se plantea que todo municipio elaborará un PADEM, debiendo considerar a lo menos ${ }^{4}$ : (i) un diagnóstico, (ii) proyecciones de matrícula, (III) metas comunales en esta materia, (iv) la dotación docente, y (v) los gastos asociados a educación.

Chile es un país altamente centralizado en la gestión del Estado, pese a que en el año 1975 se inició un proceso de descentralización que incluyó el reordenamiento espacial del territorio, brindándoles a los naciente gobiernos regionales atribuciones de gestión territorial, con la idea de comenzar un proceso de descentralización en el país, lo que en la práctica adquirió la figura de desconcentración administrativa con centralización política (Marcel y Raczynski, 2009). El Decreto Ley N³.063/1979, facultó al gobierno nacional para traspasar servicios (como salud y educación) desde la administración central del Estado a los Municipios. Un año después, en junio de 1980, entró en vigencia el $\mathrm{DFL}^{\circ} 1-3063$, que traspasó los establecimientos educacionales y su personal a los Municipios.

El PADEM es el principal instrumento que utiliza el DAEM para planificar el accionar de la comuna en el sector. La responsabilidad de su elaboración e implementación es del Municipio, asumida por lo general por los directivos del DAEM, siendo el Alcalde -como su jefe superior- quien debe presentarlo al Concejo Municipal para su aprobación, proceso que se lleva a cabo anualmente entre mediados de septiembre a mediados de noviembre ${ }^{5}$ (MINEDUC, 2009: 8). El DAEM sistematiza la información para elaborar el PADEM, considerando, aunque no en forma vinculante, el Plan de Desarrollo Comunal (PLADECO). Además, se ha de levantar antecedentes que den cuenta de los PEI y PME de los establecimientos, y programas de mejoramiento desarrollados por el MINEDUC y otros (MINEDUC, 2005: 5).

su diseño debiese implicar un completo diagnóstico de la oferta/demanda educativa de cada establecimiento y de la comuna en su conjunto. Este ha de

\footnotetext{
${ }^{4}$ No se define un formulario estándar para ello, sino orientaciones básicas que debe contemplar.

${ }^{5}$ Fechas relevantes para efectos de su disociación con los procesos educativos, cuyos resultados suelen estar disponibles en diciembre enero.
} 
ser elaborado con participación de actores claves de la comunidad, contemplando necesidades, expectativas y problemas de la comunidad educativa. Además debe permitir identificar los problemas del sector en sus aspectos técnico -pedagógicos y administrativofinancieros, con la finalidad de fundamentar las propuestas de mitigación/solución respectivas.

A partir del diagnóstico se han de desprender los objetivos y metas que respondan a las problemáticas detectadas. En esta fase el DAEM propone acciones por cada establecimiento orientadas al logro de los objetivos estratégicos. Se debe detallar el personal que requiere para su funcionamiento en docen$\mathrm{cia}^{6}$, apoyo docente, docencia- directiva y técnicopedagógica. En razón de ello, se elabora el presupuesto anual del PADEM.

La fase siguiente, de ejecución y monitoreo, implica la puesta en marcha de los programas previstos. Requiere coordinar las actividades con los responsables de cada establecimiento para alcanzar los objetivos.

\section{El PADEM debiese contener:}

- El diagnóstico de cada establecimiento escolar, en lo académico, extraescolar y administrativo.

- La oferta/demanda de matrícula de la comuna: evaluando matrícula y asistencia media deseada y esperada en los establecimientos (por su impacto en el financiamiento).

- Las metas que el DAEM y cada establecimiento pretende alcanzar.

- Dotación docente y personal no docente por establecimiento y horas totales de trabajo.

- Los programas a desarrollar durante el año en cada establecimiento y en la comuna.

- El presupuesto de ingresos, gastos e inversión para la ejecución del plan en cada establecimiento y en conjunto con la comuna.

El Alcalde presenta el PADEM al Concejo Municipal para su debate y sanción. El Plan debiese ser el instrumento de planificación estratégica por excelencia de la educación en el territorio. Por ello sus características se asocian a esa lógica, pero con un

\footnotetext{
${ }^{6}$ Este aspecto es clave pues el Estatuto Docente regula esta materia. Mediante el PADEM se puede desafectar a profesores que pertenezcan a la planta, con las cláusulas legales que ello implica.
}

ciclo operativo anual y sin tener -necesariamente- un marco de orientaciones de mediano plazo.

\section{La Planificación Local de la Edu- cación: El PADEM y el Plan Es- tratégico de los SLE}

El análisis del PADEM se construye desde la premisa fundamental que en la actualidad se requiere de procesos de planificación estratégica en la organización del Estado subnacional, en sintonía con los procesos internacionales implementados bajo una nueva visión de Estado con un rol más activo (Mazuccato, 2014), como también de las nuevas demandas que provienen de diversos campos, el económico y sus dimensiones laboral, el social y su ámbitos referidos a la construcción del pacto social, entre otros, son un aspecto crecientemente relevante para la implementación de la educación pública que requieren considerarse en su rediseño como instrumento clave de la gestión educativa local (Donoso et al., 2018).

El informe OCDE (2004), puso en alerta la gestión municipal en educación al señalar ${ }^{7}$ "...las municipalidades son muy heterogéneas. El salto desde el Ministerio al nivel Municipal, con una estructura de mandos medios que no tiene facultades para tomar decisiones parece grande y, lo que es más peligroso, desigual. Las municipales difieren en su capacidad para administrar recursos educacionales. Difieren en su interés por la educación y en la capacidad para identificar problemas y soluciones de diseño" (OCDE, 2004: 280). Proponiendo "... que se fortalezcan las estructuras medias de manera que las funciones mencionadas del Ministerio puedan ser cumplidas" (OCDE, 2004: 281).

Los DAEM conforman realidades altamente dispares. Un $65 \%$ dispone de 8 o menos establecientes escolares, con una matrícula total inferior a los 2 mil estudiantes, gran parte de ellos de enseñanza básica y con cuerpos docentes de 70 o menos educadores. En tanto, en el otro extremo, un reducido número de comunas $(15 \%)$ posee sistemas públicos con más de 15 mil estudiantes y 800 docentes aproximadamente (SINIM, 2017). No obstante estas diferencias, sus responsabilidades en el proceso educativo son las mismas.

En sintonía con ello, al tenor de los estudios en

\footnotetext{
${ }^{7}$ De los 346 municipios, más de 200 tienen menos de 2500 estudiantes, unos 40 poseen más de 10000 (hasta 40 mil aprox.), lo que demuestra la desigualdad de tamaños y realidades del sector SINIM, 2016).
} 
este plano, el PADEM no ha sido capaz de mostrar su eficiencia e impacto en la calidad de la educación, como lo refiere Raczynski y Salinas (2009):

\begin{abstract}
Aunque los PADEM casi siempre plantean una visión general que enmarcaría la administración/gestión de la educación municipal, en un alto porcentaje de municipios esta visión no se traduce en metas estratégicas ni en plazos estimados para lograrlas y no es evidente el cómo y por qué las inversiones y actividades que enuncia el PADEM contribuirán a acercar la educación municipal de la comuna al horizonte deseado.
\end{abstract}

(Raczynski y Salinas, 2009: 148).

Consistentemente se le define por algunos responsables de su diseño y ejecución como un instrumento formal de trabajo sin mayor relevancia en la operación de la comuna (Saavedra, 2009: 94), concepto ratificado por Oyarzo (2012), al señalar que las autoridades comunales cumplían con esta tarea y luego prescindían de sus orientaciones. Algunos planteamientos de (Raczynski y Salinas, 2009: 182-183) al respecto van en esta línea:

i) Todo PADEM requiere de un diagnóstico de la realidad educacional de la comuna. Sin embargo, estos tienden a ser muy generales, toman a las comunas como todos indiferenciados, hacen un manejo muy básico de la información disponible y en pocos casos cuentan con diagnósticos detallados por establecimiento. (ii) El PADEM plantea metas anuales de gestión en distintas áreas. Los proyectos de inversión que lista el PADEM son preferentemente de infraestructura, equipamiento y actividades extraescolares. Sin embargo, por lo general, las metas tienen pobres indicadores y mecanismos de seguimiento y evaluación de sus logros. (iii) Diseño de objetivos y metas poco realistas (...). iv) Todo esto hace que las metas no cumplidas de un año sean traspasadas iguales hacia el próximo PADEM (...).

Tales debilidades son coherentes con la institucionalidad municipal, no obstante los esfuerzos por fortalecer las capacidades de gestión de las áreas de educación de los municipios. En general se presenta una débil imagen pública de la administración municipal que enfrenta al dilema de consolidarse como órgano impulsor de gobierno local en esta área (Zamorano, 2006).

El PADEM al ser un instrumento de gestión anual responde fundamentalmente a una mirada de corto plazo del sector No obstante, en un alto porcentaje de municipios esta perspectiva no se traduce en metas estratégicas ni en plazos adecuados para lograrlas. Por otra parte, tampoco queda claramente evidenciado cómo y por qué las inversiones y actividades señaladas en este instrumento conducirán a la educación municipal de la comuna hacia los resultados deseados.

El análisis de planificación estratégica en el plano local de la educación, desde 1980 a la fecha, ha estado esencialmente centrado sobre los establecimientos educacionales más que en las unidades locales ${ }^{8}$, un ejemplo de ello es precisamente lo tardío de la instalación del PADEM y que el mismo Ministerio de Educación centre su accionar sobre los Proyectos de Mejoramiento Educativo de cada establecimiento, más que en el PADEM, generando un instructivo sobre el tema el año 2009 (MINEDUC, 2009) e instalando un procedimiento: "ficha del servicio educativo comunal" desde el año 2016, que esencialmente tiene un sentido formal (MINEDUC, 2016, 2017)

De esta manera la visión estratégica no ha sido el eje de la planificación local en educación, pese a que siguiendo a Armijo (2009) "Cuando hablamos de planificación estratégica nos estamos refiriendo a las grandes decisiones, al establecimiento de los Objetivos Estratégicos que permiten materializar la Misión y la Visión" (p.8).

La planificación estratégica en el ámbito público es una herramienta que nos ayudará al establecimiento de prioridades, objetivos y estrategias como apoyo a la definición de los recursos que necesitamos para lograr los resultados esperados, por lo tanto debe ser un proceso simple e incorporado en la rutina de la toma de decisiones directivas en el proceso presupuestario. Desde esta perspectiva, debemos contar con estándares de confiabilidad para identificar aspectos claves que apoyen la gestión organizacional.

(Armijo, 2009: 11).

${ }^{8}$ Ello se refuerza con los estemas de financiamiento que se han implementado, que se sustenta en el establecimiento escolar y no en la unidad local de gestión, salvo en algunos componentes en este plano de los SLE. 
De acuerdo con citeRumelt2011), lo central del trabajo estratégico es descubrir los factores cruciales de una situación y diseñar un camino para coordinar las acciones destinadas a enfrentarles.

Con posterioridad a las exigencias del PADEM planteadas en la ley mencionada, se realizaron algunas modificaciones bajo dictámenes de la Contraloría General de la República. Los dos primeros dictámenes regulan la dotación docente, pues la ley solo planteaba que en este instrumento se definiría la dotación docente, pero dejaba cierta libertad en la asignación de horas por los sostenedores, por lo cual estos dos dictámenes, estipulan que la asignación horaria debe ser ajustada a las necesidades de los establecimientos, como se detalla en un documento de contraloría:

“(...)la dotación docente se correlacione con las reales necesidades educativas de una comuna, vinculada con su número de alumnos, niveles, cursos, y planteles, entre otros; y, por lo tanto, se trata de un instrumento flexible-refiriéndose al PADEM- que debe considerar los cambios de este tipo que se experimenten durante su vigencia."

(Dictámenes $\mathrm{N}^{\circ}$ s. 57.926, de 2009, y 22.737, de 2011)

Inicialmente, y como lo define la ley $\mathrm{N}^{\circ} 19.410$, el PADEM debía ser puesto en conocimiento del Consejo Económico y Social; y remitido a los Departamentos Provinciales de Educación y a los Establecimientos Educacionales de la comuna, los que tenían un plazo de 15 días para efectuar observaciones, que debían ser acompañados para la consideración del Concejo al momento de aprobar el plan, estas exigencias fueron eliminadas (Dictámenes $\mathrm{N}^{\circ} \mathrm{s} .18 .967$, de 2012 y 32.040, de 2013).

Respecto del Plan Estratégico que deben formular los Servicios Locales de Educación -SLE- (ley $\mathrm{N}^{\circ} 21.040$, artículos 45 y 46 ) éstos establecen en su esencia:

Artículo 45.- Plan Estratégico Local de Educación Pública ${ }^{9}$. Cada Servicio Local deberá contar con un Plan Estratégico Local de Educación Pública (...), cuyo objeto será el desarrollo de la educación pública y la mejora permanente de la calidad de ésta en el territorio respectivo, mediante el establecimiento

\footnotetext{
${ }^{9}$ Texto editado de su versión original dado lo extenso del articulado (45 y 46 ).
}

de objetivos, prioridades y acciones para lograr dicho propósito. Será elaborado por el Director Ejecutivo y aprobado por el Comité Directivo Local, y tendrá una duración de seis años desde su aprobación.

El Director Ejecutivo deberá presentar una propuesta de Plan seis meses antes del término de la vigencia del Plan anterior, y considerará los niveles educativos, formaciones diferenciadas, modalidades educativas $\mathrm{y}$ contextos de la oferta educativa del territorio. El Plan deberá contener, al menos, lo siguiente:

1. Diagnóstico del servicio educacional del SLE en el territorio, con especial énfasis en las características de los estudiantes y en la situación de los establecimientos.

2. Objetivos y prioridades de desarrollo de la educación pública en el territorio a mediano plazo. Estos objetivos deberán ser concordantes con los establecidos en el convenio de gestión educacional y en la Estrategia Nacional de Educación Pública.

3. Para la elaboración y modificación del Plan se considerarán los siguientes elementos (La Estrategia Nacional de Educación Pública, regulada en el artículo 6; La Estrategia Regional de Desarrollo, de acuerdo a lo contemplado en el decreto con fuerza de ley $\mathrm{N}^{\circ} 1$, de 2005 , del Ministerio del Interior. Los PEI y PME de los establecimientos educacionales de su dependencia.

Para elaborar la propuesta de Plan, el Director Ejecutivo deberá consultar al Consejo Local, el que podrá formular recomendaciones. Asimismo, deberá solicitar la opinión de los directores de los establecimientos del territorio.

La propuesta del Plan deberá ser aprobada por el Comité Directivo Local, el que podrá hacer observaciones y proponer modificaciones por razones fundadas en lo dispuesto en los incisos tercero y cuarto. El Director Ejecutivo podrá incorporar las observaciones planteadas por el Comité Directivo Local o mantener su propuesta, indicando las razones que la sustentan, remitiéndola a ese Comité para su decisión.

El Plan podrá modificarse por cambios sustantivos en los contenidos dispuestos en el inciso tercero, por fuerza mayor o por caso fortuito. La aprobación de dichas modificaciones deberá seguir las mismas formalidades establecidas 
en el presente artículo.

En el artículo siguiente ( $\mathrm{N}^{\mathrm{O}}$ 46) se especifica el Plan Anual y se relación con el Plan Estratégico. El Director Ejecutivo presentará al Comité Directivo Local y al Consejo Local, a más tardar el 15 de octubre de cada año, un plan anual para el año siguiente que deberá contener, a lo menos, los siguientes elementos:

1. Estado de avance de objetivos y metas contenidas en el convenio de gestión educacional, así como los contenidos en el plan estratégico local y los proyectos educativos institucionales de cada establecimiento de dependencia del Servicio Local.

2. Dotación de docentes y asistentes de la educación para el ejercicio de las funciones administrativas y pedagógicas para el desarrollo del proyecto educativo institucional, , en cada establecimiento educacional de dependencia del Servicio Local, la que deberá fundarse en razones técnico-pedagógicas y determinarse sobre la base de, al menos, los siguientes elementos:

a) Matrícula total de cada establecimiento.

b) Niveles y modalidades de la educación provista por cada uno de éstos.

c) Plan de estudios de cada uno de ellos o proyecto educativo institucional en el caso de la educación parvularia.

d) Componentes de los PME elaborados con la comunidad de cada establecimiento, de conformidad a lo establecido en la ley $\mathrm{N}^{\circ}$ 20.248, sobre SEP, y los proyectos de integración escolar vigentes de dichos establecimientos que tengan relación directa con sus requerimientos de dotación de docentes y asistentes de la educación.

3. Acciones de apoyo técnico-pedagógico de cada uno de los establecimientos educacionales de dependencia del Servicio, determinando la periodicidad y contenidos generales de éstas. La planificación y ejecución de dichas acciones considerará el plan estratégico del Servicio. Para su elaboración, el Director Ejecutivo consultará a los equipos directivos de los respectivos establecimientos educacionales, teniendo en consideración las acciones definidas en los planes de mejoramiento educativo de éstos y en los convenios de desempeño de cada director de establecimiento educacional. Una vez presentado el
Plan Anual, el Comité Directivo Local y el Consejo Local de Educación contarán con un plazo de quince días hábiles para realizar recomendaciones.

El Director Ejecutivo sancionará el plan a más tardar el 15 de diciembre de cada año, el cual deberá ajustarse a los recursos y dotaciones totales de docentes y asistentes de la educación del Servicio Local, definidos por la Ley de Presupuestos del Sector Público para el año siguiente.

Como se observa ambos instrumentos -uno desde su práctica, otro en sus normas- poseen elementos comunes sobre sus procedimientos, contenidos, como también algunas orientaciones compartidas, hay diferencias respecto de su extensión -uno es anual y otro sexenal con un ajuste anual-, y con plazos calendario diferentes para su gestión, pero no hay -al menos hasta el momento-, caminos claros de vinculación con los otros instrumentos: PLADECO, PEI y PME de los establecimientos, más allá de su mención, tampoco respecto de los indicadores derivables del diagnóstico, objetivo -metas, materias claves que muestran la necesidad de profundizar en las mejorías en este plano, toda vez que estas -eventuales debilidades- son compartidas en el diseño de ambos procesos.

Como señalan Raczynski y Salinas (2009), y como también se deriva de los resultados que se exponen más adelante, no hay a la fecha vínculos claros entre el PADEM y el mejoramiento educativo en nuestro medio, cuestión fundamental que Bryk et al. (2010), evidencian la complejidad de poder establecer estas relaciones, las condiciones bajo las cuales se producen para generar impactos, mostrando la distancia que tenemos en este campo, donde la política local ha variado regularmente existiendo baja continuidad, recursos financieros inadecuados y una esta débil y dependiente estructura orgánica con excesiva dependencia del Alcalde, materias que Anderson, citeMascall2012 enfatizan, mostrando los diversos enfoques para alcanzar impactos de mediano plazo, y las exigencias que deben instalarse en el plano organizacional para impactar en el mejoramiento desde la política local, los cuales no se cumplen en el plano municipal, y en el caso de los SLE es un tema por verse. Materias que en el caso en análisis distan notablemente de los procesos de planificación estratégica local de los territorios que han tenido experiencias exitosas en este plano (Leithwood y Azah, 2017).

El análisis de esta problemática se ha enfocado desde la visión de evaluación de los instrumentos de 
política educativa sustentada desde el enfoque del "Ciclo de las Políticas" de Bowe et al. (1992), relevando la naturaleza compleja de la política educacional y estableciendo un modelo analítico para identificar el proceso de implementación de políticas. La atención por la política pública y la política educacional es parte del pensamiento de Ball (1993) surge de conceptualizar el proceso de formulación de políticas como: (i) el contexto de influencia, (ii) el de la producción del contenido de la política y, (iii) el contexto de la práctica, siendo tales contextos cíclicos (por ello, el análisis cíclico de la política). Su dinámica no implica seguir ese orden. El mismo Ball (2015) actualiza su propuesta remarcando que las políticas son mediadas y diferentemente representadas por distintos actores en contextos diversos, aunque al mismo tiempo son producidas y formuladas por conocimientos y premisas tomadas como ciertas e implícitas sobre el tema y sus actores.

Complementando esta visión, Mainardes (2006) sostiene que los pensamientos de Ball "... rechazan los modelos de política educacional que separan las fases de formulación e implementación porque ignoran las disputas sobre la política" (2006: 49). Los autores perfeccionan el modelo de ciclo de políticas proponiendo un "ciclo continuo", sumando al modelo un cuarto y quinto contexto: el de resultados/efectos y las estrategias. El contexto de influencia se refiere al momento en que las políticas se discuten y se construyen (Mainardes, 2006: 51).

\section{Consideraciones metodológicas}

Se trata de un estudio exploratorio/descriptivo de carácter cualitativo, sustentado en entrevistas semi estructuras de actores claves relacionados con el diseño e implementación del PADEM, la cuales se realizaron durante el cuarto trimestre del año 2017, a Jefes DAEM que tienen responsabilidad y experiencia relevante en el diseño y ejecución del PADEM.

Los veinte (20) participantes entrevistados son profesionales que ejercieron o ejercen el cargo de Jefe DAEM en comunas de la Región del Maule (territorio central de Chile), cuya experiencia en el cargo va de 5 a 20 años de ejercicio. Su selección fue intencionada, en tanto cumpliendo los requisitos estaban dispuestos a participar

Este grupo representa el $67 \%$ de quienes ocupan el cargo en la región y el 5,8\% del universo total país El estudio no tiene aspiraciones representativas sino de profundización en un tema insuficientemente estudiado en términos de au impacto y proyección.

La operacionalización de las variables corresponde a la estructura etápica que se define para desarrollar un PADEM por las normas en este plano, y las preguntas fueron generadas en función de identificar los procesos seguidos y sus problemas, Estas interrogante fueron validadas en dos oportunidades con grupos equivalentes (4 casos en cada etapa -ex jefes DAEM con desempeño de al menos 4 años en el cargo), los que no fueron considerados en el estudio final.

El guión de entrevistas fue validado siguiendo para cada pregunta los criterios de pertenencia, relevancia, comprensión del enunciado y aporte a los objetivos de la investigación, mediante una pauta que se le entregó a cada uno que debía puntuar de 1 a 4 cada pregunta (1 no pariente - 4 muy pertinente), así como realizar al sugerencias de contenido redacción que estimara adecuadas. El análisis de datos sigue las etapas definidas y se organizan las respuestas en función de las frecuencias registradas por cada una. Las preguntas de las entrevistas se asocian con cada categoría, a saber:

Tabla 1: Operacionalización de las variables Categoría Subcategoría

\begin{tabular}{ll}
\hline Elaboración & $\begin{array}{l}\text { Elementos importantes } \\
\text { Dificultades del diseño }\end{array}$ \\
\hline $\begin{array}{l}\text { Implementación } \\
\text { y seguimiento }\end{array}$ & $\begin{array}{l}\text { Dificultades implementación } \\
\text { Seguimiento } \\
\text { Dificultades seguimiento }\end{array}$ \\
\hline $\begin{array}{l}\text { Aportes a la } \\
\text { gestión municipal }\end{array}$ & $\begin{array}{l}\text { Aportes } \\
\text { Vinculación con el PEI }\end{array}$ \\
\hline $\begin{array}{l}\text { Modificaciones al } \\
\text { PADEM }\end{array}$ & Modificaciones al PADEM \\
\hline
\end{tabular}

Fuente: Elaboración propia.

La primera fase de análisis, a saber diagnóstico, implicó precisar la relevancia de la etapa, sus principales componentes y las dificultades experimentadas en esta fase. La segunda categoría, a saber, implementación y seguimiento, comprende identificar el concepto, y luego los problemas que se han experimentado al respecto en materia de implementación y análisis de resultados. La tercera dimensión busca identificar cómo se visualiza el PADEM respecto del aporte a la gestión local del sector, y finalmente, precisar las sugerencias de modificaciones al PADEM en términos conceptuales, de contenido, operacional y metodológico. 


\section{Resultados del proceso de entre- vistas a acores claves}

\subsection{La fase de elaboración del PADEM}

El análisis de esta etapa se desglosa en dos dimensiones: (i) los componentes más relevantes del proceso y su jerarquización; y (ii) las principales dificultades/ problemas que se enfrentan en esta fase. Los entrevistados reconocen en forma unánime que la fase de "diagnóstico" es fundamental, generándose información relevante y actualizada sobre aspectos claves de cada establecimiento y del territorio:

[...]para un buen diagnóstico hay que llegar a personas claves, para levantar las necesidades, pero hay que canalizarlas porque tienen que estar acordes al proceso administrativo.

(Entrevistado 5)

Idealmente un buen diagnóstico se construye desde variables estratégicas, debe realizarse desde la reflexión y participación de los agentes educativos, para la validación de la actualización del diagnóstico de cada establecimiento.

(Entrevistado 9)

El segundo componente es la traducción de las principales necesidades en la oferta/ demanda docente, a saber, la dotación docente:

Una parte fundamental es la dotación docente, las horas que le asigne a cada profesional para cumplir con las necesidades educativas.

(Entrevistado 6)

La dotación docente tiene que planearse con rigurosidad para establecer los egresos de la forma más fidedigna posible.

(Entrevistado 8)

El factor siguiente es el presupuesto disponible, entendido como el balance entre la proyección de recursos requeridos y los posibles de obtener ${ }^{10}$, las brechas que se generan y la forma de financiarlas, lo que corresponde al debate del PADEM con los Concejales. Algunas respuestas fueron:

\footnotetext{
${ }^{10}$ En el sistema de financiamiento público escolar, el Estado no asume el financiamiento de toda la operación del establecimiento, sino que entrega subsidios con los cuales éste debe operar, ajustándose a esos montos.
}

Una clave es tener un PADEM financiado, y que se sustente en un ordenamiento de horas y de una muy buena distribución de las subvenciones.

(Entrevistado 8)

Debe tenerse claridad absoluta de la situación financiera (...) de tal manera que los recursos económicos estén para cubrir las necesidades.

(Entrevistado 1)

La jerarquización de los componentes es indispensable para comprender la lógica del PADEM, y su dominio por los entrevistados. Fue consensuado que el diagnóstico es clave, como lo es su traducción en términos de la oferta pedagógica del Municipio (dotación docente), y por ello la estructuración presupuestaria para determinar las brechas en este plano y generar los requerimientos para definir el origen de los recursos que concretarán la implementación.

El segundo componente de esta sección se refiere a las principales dificultades de la fase de diseño. Las respuestas dominantes (la mitad de los entrevistados -10) se refieren a los "plazos considerados":

(...) los plazos son mínimos y la participación cuesta coordinarla, para que estemos todos abocados al PADEM, los quehaceres, las actividades. Los compromisos de los establecimientos dificultan destinar tiempo a este instrumento.

(Entrevistado 2)

Sabiendo que el diagnóstico debiese ser una fase de mayor esmero. Debiera ser más profundo, pero se hacen a la rápida y a veces sólo por cumplir.

(Entrevistado 15)

En segundo lugar, seis entrevistados coinciden en que "el respaldo político" es una dificultad o factor determinante en el proceso de elaboración del PADEM:

Las principales dificultades son las que va imponiendo el Alcalde con el Consejo, pues se requiere de su aprobación. Ellos tienen una mirada política.

(Entrevistado 1) 
Si las autoridades políticas no apoyan no tenemos PADEM, por ende, es fundamental su apoyo.

(Entrevistado 14)

En esta fase, las dificultades más relevantes se sitúan en dos planos. Primero en lo técnico, los plazos para levantar, procesar y analizar la información y proponer un Plan sustentable. Componente que tiene tres acepciones, (i) lo breve del tiempo en referencia a la extensión y complejidad de las tareas; (ii) la relación entre la fase de diseño del PADEM y el desarrollo del calendario escolar. Finalmente, (iii) la visión cortoplacista del sistema anual de trabajo del PADEM, mencionado también por Raczynski y Salinas (2009) y González et al. (2015).

En el segundo plano destaca el respaldo político. En este caso queda en evidencia por los entrevistados, los criterios no educacionales que consideran las autoridades para evaluar y decidir sobre las acciones. Este argumento es consistente con la importancia de desalcaldizar la educación ${ }^{11}$ y traspasarla a una entidad técnica que pueda superar esta limitante.

\subsection{Análisis de la fase de Implementación y seguimiento}

Esta fase considera las (i) dificultades recurrentes de implementación; (ii) su seguimiento; y (iii) sus problemas/ dificultades más relevantes. Respecto de las complejidades de implementación es consensuado que los recursos económicos son un referente obligado y decisivo (16 entrevistados):

Quiero minimizar lo financiero porque los recursos están. Hay que saber administrarlos, y aquí no hay mucho que analizar porque todos los recursos tienen que estar a disposición del tema educacional.

(Entrevistado 2)
En la implementación a veces faltan los recursos porque se ha invertido más o se ha gastado en cosas que no aportan a la educación, a veces las municipalidades contratan gente en las unidades educativas, paradocentes, no docentes, orientadores, etc. que el dinero que llega vía subvención no nos alcanza.

(Entrevistado 8)

Los entrevistados ratifican la importancia de los recursos financieros, precisando las dificultad de sincronizarles con los plazos y requerimientos pedagógicos, las limitaciones del sistema de compras púbicas, y que su uso sea para cuestiones sustantivas.

Un segundo aspecto se asocia con la capacidad de los equipos técnicos de los DAEM para desempenar estas tareas. Se manifiestan diferencias entre los DAEM de comunas grandes (unos 40 en el país ${ }^{12}$ ) de los otros 200 de unidades muy pequeñas y en el intermedio otros tantos -100- con personal variable, por ende, el tema sustantivo es disponer de personal profesional competente para las tareas:

En la parte pedagógica hay que insistir, porque tenemos una unidad pedagógica muy pequeña, no hay evaluador, no hay orientador, no hay curriculista, entonces, a lo mejor tener un mejor equipo de unidad técnica.

(Entrevistado 4)

Se refiere a las capacidades de los líderes del proceso educativo en el aula: Una dificultad a nivel técnico, pasa por las capacidades de los profesores, faltan competencias docentes, como las de antaño, hemos descendido como profesionales. Eso dificulta a los profesores entregar el conocimiento a las personas.

(Entrevistado 12)

El segundo factor es la posibilidad de disponer del personal técnico suficiente y capacitado para asumir tareas más complejas en este ámbito. Ello es determinante, y es una herencia del diseño de la reforma de 1980 que estableció que los temas técnicopedagógicos eran del Ministerio sectorial, cuestión que cambió cuando ya los municipios habían experimentado reducciones significativas de sus ingresos,

\footnotetext{
${ }^{12}$ Nos referimos a tener más de 10 mil estudiantes. La propuesta de nuevos SLE parte configurando territorios con 8 mil estudiantes y más, ello tiene incidencia en los recursos financieros para poder constituir equipos técnicos comunales que den soporte a esta tarea, aspecto crítico en los territorios pequeños.

\footnotetext{
${ }^{11} \mathrm{El}$ concepto "desalcaldizar" responde al hecho que la crisis no solo se debe a la gestión del municipio, sino a la excesiva capacidad de intervención que posee la autoridad política del municipio, por ende no es lo mismo desmunicipalizar que desalcaldizar.
} 
producto de la pérdida sostenida de matrícula, por lo que solamente los DAEM de las ciudades grandes pudieron implementar equipos técnicos más completos.

Respecto del seguimiento del PADEM, las respuestas señalan en igual proporción que se efectúa como que no se realiza seguimiento de las principales acciones:

el seguimiento del PADEM lo hacemos con una unidad que está dentro del departamento de educación (Unidad de Control de Gestión). (Entrevistado 2)

una unidad técnica es la encargada de monitorear, tienen pautas para ir cerciorándose que se lleve a cabo.

(Entrevistado 1)

hacíamos responsables al jefe de la unidad. Eso permitía después cotejar el avance de la implementación del PADEM, poder hacer una evaluación, que la hacíamos semestral ya que es mucho el trabajo al interior del departamento. (Entrevistado 8)

Por otro lado, la mitad de las respuestas se agrupan en la categoría "sin seguimiento":

No hay seguimiento, porque no hay evaluación, sino solamente saber si se hizo o no.

(Entrevistado 5)

La debilidad es no contar con instrumentos que pudiéramos decir, esto es una rúbrica, un formato en el cuál vamos a poder hacer un seguimiento.

(Entrevistado 11)

Aquí no tenemos nada, formalmente no existe, así que efectivamente estamos al debe.

(Entrevistado 20)

Al profundizar las dificultades del seguimiento se reitera el factor "tiempo disponible" para efectuar las tareas y la fase del año en que se elabora, procesa y evalúa el PADEM, y la falta de sincronía con los tiempos educativos, Igualmente, la idoneidad del personal disponible es recurrente, y en un grado menor, la intervención de la autoridad política en la tarea educativa.
Entre los factores más destacados está la necesidad de disponer de indicadores de seguimiento (proceso/producto) que sean relevantes para el componente educacional, y que permitan adoptar medidas correctoras oportunas. Esto implica sincronizarles con el proceso educacional, diseñándose un instrumento ágil, centrado sobre aspectos críticos que permitan adoptar medidas pertinentes y oportunas. Ello ratifica como uno de los problemas sustantivos de gestión de nivel comunal la precaria institucionalidad que además se concentrar en las tareas de gestión administrativa (Donoso y Benavides, 2017).

\subsection{Principales aportes del PADEM a la gestión comunal de la educación}

El positivo que el PADEM sea un instrumento validado, no obstante, el reconocimiento que goza es esencialmente genérico, a saber, permite una visión amplia, lo que es coherente con lo expuesto en la revisión de la literatura. En segunda instancia se indaga sobre la vinculación con los otros instrumentos de planificación a nivel de establecimiento: Proyecto Educativo Institucional (PEI) y Programa de Mejoramiento Educativo (PME), las respuestas señalan:

Los PEI parten generando sus propios objetivos y metas. Son quienes conducen los PEI, sea los consejos escolares o directivos de los colegios, los que levantan las propuestas para el PADEM.

(Entrevistado 2)

En rigor los PEI debiesen dar origen al PADEM, es decir, los PEI son interpretados por el PADEM.

(Entrevistado 13)

Creo que más que el PADEM, la carta de navegación es el PEI, porque el PADEM se veía como anexo, como que había que cumplir por ley, porque nunca se vio el real efecto de un PADEM, en las escuelas.

(Entrevistado 18)

Otras respuestas aluden al aporte general del DAEM a la gestión:

Es un instrumento de planificación estratégica para el sistema educativo comunal (...).

(Entrevistado 4) 
El PADEM sirve como guía, como instrumento orientador y carta de navegación, por lo tanto, te facilita la gestión.

(Entrevistado 6)

Si bien se señala la lógica de operación, PEIPADEM, en los hechos no se identifican plenamente los conectores entre ambos instrumentos, más allá del que hacen los responsables de los establecimientos escolares, aludiendo a que poseen racionales diferentes. De hecho, un par de entrevistados señaló que no existen vínculos claros:

Debieran vincularse. Pero no hay certeza, porque no existiendo el seguimiento va a ser difícil ver cómo hablamos el mismo idioma desde el PADEM, que es la política municipal, cuál es el PEI y yo incorporaría además al PME. (Entrevistado 9)

Por ese lado va la tarea pendiente: tenemos que vincularlo.

(Entrevistado 3)

En síntesis, se trata de una dimensión que requiere de una operacionalización mucho más desarrollada y estructurada, que permite al articulación de los instrumentos y su vinculación ágil y oportuna, para aprovechar sus eventuales potencialidades. La vinculación planificación- mejoramiento continuo es débil, esta relación es un factor más que clave, en razón de ello una propuesta de mejoramiento en este plano debe abordar, con una metodología que permita vincular los procesos y su resultados en los periodos considerados, a fin de determinar su impacto en el mejoramiento educativo..

\subsection{Propuestas de cambios o ajustes al PA- DEM}

Las propuestas al respecto se organizan en dos secciones: (i) consideraciones técnicas respecto del instrumento, y (ii) aquellas de contenido y políticas.

\subsubsection{Propuestas sobre ámbitos técnicos del ins- trumento}

Un aspecto que destaca es el tiempo/plazo y su sincronía con los procesos educacionales. Lo central es que el PADEM es un Instrumento diseñado con la lógica de la gestión presupuestaria anual pública, que se presenta en septiembre de cada año para su aprobación en noviembre y ejecución a partir de enero del año siguiente. Esta lógica/plazos no es la del hacer educativo, que tiene un año calendario de marzo a enero. Por ende, la planificación local de la educación no debiese seguir con este desfase por más de dos décadas de operación, lo que implica cambios en su cronograma de diseño, seguimiento y evaluación anual, y también plazos razonables y consistentes con el calendario pedagógico, materias que inciden fuertemente en su eficacia.

Un segundo elemento lo menciona la mitad de los entrevistados: debiera planificarse a mediano plazo (multianual), y no a un año plazo.

Se debiera ajustar el tiempo de los instrumentos, ejemplo: si el PEI es a 4 años, el PME y el PADEM también lo sean, eso puede ser una innovación.

(Entrevistado 8)

Siempre he pensado que el PADEM debería ser de 4 años, lo que dura la gestión de un alcalde, porque después viene otro y no sigue con lo misma.

(Entrevistado 4)

Dado que los procesos educacionales son de lento y largo desarrollo, habría que pensar en un horizonte mínimo de 4 años con ajustes anuales, provenientes de la evaluación de los componentes medulares del Plan, este ciclo se sincronizaría con la gestión comunal que es de 4 años. En forma complementaria varios participantes (6) señalan la conveniencia de un formulario único para diseñar y evaluar el PADEM. De forma que sea claro lo que debe contener, qué indicadores son los sustantivos, cómo se miden, con qué frecuencia, etc.

Sería bueno contar con un formato, único. Un formato similar, porque en las escuelas todos hacemos prácticamente lo mismo, y también debería haber más asesoría del Ministerio de Educación.

(Entrevistado 5)

Utilizar un formato único es una ayuda técnica si se quiere dar un mejor uso del mismo. Implicaría una revisión detallada de la metodología para cada fase. Hay experiencias en este plano en otras áreas que son interesantes.

(Entrevistado 17) 
El proceso de seguimiento del Plan fue mencionado desde el inicio como central y sobre el que los participantes, unánimemente, indican la necesidad de mejoras técnicas: disponiendo del tiempo y personal capacitado para implementarle, de variables/indicadores claves, relevantes pedagógicamente, simples de medir y seguir; y con un sistema definido de seguimiento político del mismo.

En términos técnicos, la falta de tiempo. Para hacer este seguimiento tomando en cuenta toda la sobrecarga administrativa que tenemos en el departamento, los tiempos eran bastante acotados. Creo que es una debilidad, no sé si serán los tiempos y el poco personal competente para poder hacer esto.

(Entrevistado 8)

Cuatro entrevistados indican además que hay que mejorar el apoyo técnico del Ministerio:

Que las unidades fueran capacitadas en este modelo que queremos implementar, con las partes que debe tener el PADEM, debe haber elementos centrales que han de estar presentes.

(Entrevistado 1)

Lo fundamental es cómo trabajamos con el Ministerio de Educación, podemos hacer aportes, que sea ley. Que los alcaldes que no cumplan sean sancionados sus jefes comunales.

(Entrevistado 3)

En razón de lo expuesto destaca que técnicamente el PADEM requiere reestructurarse desde la visión pedagógica de la educación local pública. Salir del enfoque de gestión pública convencional y considerar el sentido y plazos de las tareas educativas. Entonces, debiese revisarse los tiempos educativos, los plazos para diagnosticar, adecuar el sistema de indicadores a las tareas más significativas, impactos reales del trabajo de los establecimientos y de la comuna.

En segundo lugar, sería importante alinear sinérgicamente los instrumentos y que se instale un sistema de seguimiento sobre hitos claves del año escolar, sustentados en indicadores relevantes que den cuenta -oportunamente- del proceso para realizar las correcciones debidas en lo s momentos que corresponda.

\subsubsection{Propuestas sobre los elementos de contenido y politicos del mismo}

Un elemento estratégico en esta materia es cambiar o adecuar el "Proceso de aprobación", con opiniones como:

(...) y también la parte del consejo municipal, no estoy de acuerdo que sean ellos quienes aprueben el PADEM, porque no tienen mucha idea de educación. (...) la educación jamás debió haber pasado a las municipalidades.

(Entrevistado 4)

Es burocrático que pase por el Consejo, yo buscaría esa línea del consejo ¿porque no es el ente idóneo? - es que dos o tres personas son los que realmente te hacen los aportes reales, entonces sientes que estás hablándole al viento.

(Entrevistado 5)

Un segundo elemento es que el Concejo no realiza un seguimiento del PADEM tras su aprobación. Por ende, la aceptación de los representantes ciudadanos tiene un vacío que es importante y que remarca, el poder asimétrico del Alcalde, usualmente con consecuencias no siempre positivas para la educación Por ende, el seguimiento del PADEM desde lo político requiere de cambios en el accionar de las unidades territoriales del Ministerio y de la comuna, pues éste no dispone de mayores atribuciones para el seguimiento eficiente de metas y compromisos.

Entre otras dimensiones políticas, con menor frecuencia que las anteriores, están las opiniones de la mitad de la muestra (9), las cuales se refieren al "Estatuto Docente":

El estatuto es un tema complicado porque hay profesores con titularidad, pero las escuelas van cerrando y hay que trasladarlo, porque uno podría finiquitarlo pero tampoco está el dinero para ello, es un tema importante en el PADEM.

(Entrevistado 6)

... tenemos muchas problemáticas en cómo mejorar el proceso de asignación de horas, hay docentes que a veces adquieren titularidad y no tienen las competencias ni preparación o especialización que le permita hacerse cargo de un nivel de una asignatura que pudiésemos tener 
mejores resultados. Hay que hacer un trabajo muy intenso de personal, en la dotación docente.

(Entrevistado 2)

Finalmente, seis participantes señalan la importancia de la participación:

... que los consejos escolares fueran parte para que vean como se hace esto, yo eso lo cambiaria, que lo hiciera más gente esto, juntarse con los establecimientos con los apoderados para que ellos sean parte.

(Entrevistado 6)

Encuentro que mientras más gente se una al equipo técnico del PADEM, se hace más fuerte, el mismo El Consejo debería ser ente asesor, y de ahí sería un aporte, porque ellos tienen la visión de cada punto de la comunidad.

(Entrevistado 5)

El seguimiento técnico y político del PADEM es un factor clave en el amejoramiento de la educación local y requiere de un sistema entallado que provea datos confiables para los indicadores determinados y cuya sustantividad alimente en forma oportuna la toma de decisiones que debe tener un fuerte sustrato técnico, y también una supervisión política, esto es que ciudadanos y autoridades, en procesos regulados tomen conocimiento del progreso seguido y atiendan la sugerencias de rectificación que se propongan o bien insten a su adopción de no ser debidamente implementadas.

\section{Discusión final y propuestas}

Tras veinticinco años de vigencia del PADEM, éste se encuentra debidamente integrado al quehacer del sector, siendo asumido por sus líderes sectoriales. No obstante, es complejo apreciar su impacto como herramienta de mejoramiento de la política y de la gestión, al tenor de los resultados que no han sido positivos dado que primero se integró tardíamente al hacer de los municipios en este campo. En segundo lugar, se instala en un escenario que fue diseñado para favorecer la privatización de la educación, no pudiéndose atribuir este problema solamente al PADEM, pero ciertamente instala una práctica que han terminado siendo inconducente para impulsar ciclos de política de corto plazo, cuyos impacto en términos de su aporte para enfrentar las grandes dificultades es más bien escaso. Prueba de ello son las críticas sustantivas que se han formulado sobre su diseño, seguimiento, implementación y control que demuestran la importancia de generar un nuevo instrumento que implique plazos, métodos y contenidos diferentes, a la luz de las características del ciclo formativo escolar y del ciclo de la política educativa local.

No caben muchas dudas, considerando las dificultades reseñadas, que el PADEM no ha sido el instrumento más idóneo para acometer esa tarea, adoleciendo de una visión de estratégica de políticas públicas locales y de procesos (diseño, aprobación, seguimiento, evaluación) con serias debilidades técnicas (de articulación con otros instrumentos y de relevancia de sus metas y procedimientos) como también de carácter político, derivadas de la forma como se elabora, se controla y valida sus propuestas.

Si bien no se le ha impedido a la educación pública adoptar otros instrumentos en este campo, muchos DAEM no lo han hecho dado que no poseen el personal técnico competente ni tampoco los recursos financieros para acometer tareas de mayor envergadura que permitirían una mayor proyección, es decir el contexto obliga a ceñirse a este instrumento, y por esta vía se previesen los problemas que han enfrentado. Incluso en muchos casos se han externalizado estas tareas reduciendo con ello el que queden aprendizajes instalados en el DAEM en este campo.

Ello requiere que el Estado central fortalezca el paradigma de la colaboración interinstitucional y no la competencia entre los diversos entes públicos como ha sido la tónica de los últimos cuarenta años. Lo que ciertamente implica reorientar su hacer bajo un giro paradigmático complejo de instalar dado que la sociedad funciona en todas sus esferas bajo el formato de la competencia, incluso entre el mismo Estado y sus sub-unidades territoriales. Tampoco hay un diálogo fluido entre la lógica de los diversos instrumentos de planificación comunal (PLADECOPADEM) con la de los establecimientos escolares (PEI- PME), requiriéndose de conectores más evidentes entre éstos que hoy escasamente interactúan, más por voluntad, habiendo transcurrido tiempo suficiente para hacer estos ajustes.

Es importante repensar este tema desde la política pública, asociada a los ciclos políticos de gestión de los gobiernos nacionales y locales (4 años), no solamente para simplificar este proceso, sino para ligarlo con objetivos estratégicos de gobierno del periodo de la autoridad respectiva, lo que debiese implicar un 
mayor compromiso en este plano ${ }^{13}$.

El desafío de fortalecer la educación pública también implica pensar en un rediseño sustantivo de este mecanismo de planificación local, que no implique repetir errores. Incluso a una escala mayor como son los referidos a los SLE, sino por el contrario, acometer una tarea de magnitud, pues implica articular los otros instrumentos empleados por los establecimientos escolares, en la cual Chile está en deuda desde hace tiempo.

En cuanto a los aspectos técnicos, la fase de diagnóstico es clave y presenta muchas dificultades en referencia a su asincronía con los plazos del proceso educativo, con la organización de los recursos y el presupuesto derivado de ello. En esta fase se enfrentan limitantes técnicas de los DAEM con políticas derivadas del proceso analizado y de los plazos de tramitación/sanción del PADEM, a lo que agrega la complejidad del respaldo político de las autoridades de la comuna como una limitante reiterada que merece más atención para equilibrar elmhacer ciudadano con el indispensable componente técnico.

Una segunda dimensión de las dificultades técnicas proviene del corto plazo que supone metas esencialmente anuales y la ambigüedad bajo la cual se formulan muchos propósitos, lo que implica que sean consideras reiterativamente en cada formulación del PADEM, poniendo en evidencia la necesidad de diseñar un instrumento plurianual, de actualización periódica, asentado en indicadores sustantivos, simples y que guíen oportunamente las decisiones tanto del sistema comunal como de las escuelas.

La fase de seguimiento denota dificultades para poder hacer un proceso sistemático, sea por razones de tiempo, calificación del personal, definición de indicadores pertinentes y relevantes, así como de plazos para operar. Hay un cumplimiento formal de estos aspectos, aunque no implica que el PADEM sea el documento de consulta constante que guía cada paso. Es importante relevar que los aportes del PADEM se refieren a promover una visión amplia del proceso, pero necesita de transformaciones sustantivas, de un rediseño de elementos de forma y fondo, técnicos, políticos y de contenido.

Los aportes en este plano son relevantes. Suponen diseñar un nuevo instrumento o bien una adecuación

\footnotetext{
${ }^{13} \mathrm{El}$ plazo de 6 años considerado para la gestión de los SLE si bien es una medida eficiente para saltarse el ciclo político contingente, descompromete a los actores públicos en el gobierno entrante al encontrarse con objetivos definidos sin poder adecuarles en algún grado.
}

profunda del existente, decisión que ha de evaluarse, en lo fundamental, sería importante ceñirse a las siguientes orientaciones:

1. Generar un instrumento plurianual, asociado al periodo de gobierno de las autoridades locales (regionales y nacionales). Sustentado en un diagnóstico sólido, con indicadores relevantes que contribuyan al establecimiento de metas anuales y del período, hacer un seguimiento de su evolución, brechas registradas, requerimientos de ajustes y corrección, incluyendo el ámbito presupuestario.

2. Que el proceso de elaboración sea consistente con los plazos del calendario escolar, por a importancia de contar con información sólida sobre lo ocurrido y no meramente estimaciones, para su adecuada decisión.

3. Que considere un formulario tipo, flexible, con definiciones claras, indiciadores precisos y validados (cualitativos y cuantitativos), y procesos de diseño, seguimiento y evaluación explícitos, de carácter participativo y con requerimientos y criterios técnicos bien establecidos.

4. Que sea vinculante con un diagnóstico solvente, traducido a metas consistentes y propuestas estratégicas que impliquen definir y proyectar recursos, que respeten las asignaciones presupuestarias en los tiempos pedagógicos requeridos.

5. Que su diseño y lógica de operación se articule con los otros instrumentos de los establecimientos (PME- PEI)y comunal (PLADECO), generando sistemas de indicadores integrado.

6. Que su seguimiento implique instalar instancias técnicas sustentadas en indicadores pertinentes y simples, que alimenten oportunamente las decisiones de los diversos niveles del sistema local y sus establecimientos educacionales.

7. Que el seguimiento político del mismo considere criterios técnicos y políticos solventes, conocidos y evaluados públicamente.

8. Que sus fases dispongan de los tiempos requeridos por la complejidad de la tarea, capacitándose a quienes dirigirán estos procesos y producirán los diversos documentos consignados.

9. Que la comunidad se capacite para participar constructiva y colaborativamente en este proceso. 
Respecto de la extensión de estas consideraciones a los SLE, ciertamente en términos genérales son todas aplicables, con algunas limitaciones, a saber:

1. Si bien el Plan estratégico es sexenal, sus fuentes y procedimiento de operación (normativos, operacionales y de contenido) son muy similares a los del PADEM. Se requiere de una operacionalización clara que vincule los instrumentos locales con el Plan y éste con los PME -PEI de lso establecimientos.

2. Un segundo elemento es que este diagnóstico ha de ser participativo, con la salvedad que los SLE no dependen del Municipio y del Gobierno Regional (GORE), sino de la Dirección Nacional de Educación Pública, ente centralizado. Es decir, se ha generado un proceso de recentralización de la educación pública que otorga mucho poder al Director del SLE y deja al Consejo y otros organismos -en general- en el plano de recomendaciones.

3. Se requiere precisar, al igual que en los PADEM, los hitos claves del proceso anual, los indicadores, las etapas de seguimiento, la información que debe ser reportada y las decisiones que se pueden adoptar conforme los resultados alcanzados.

4. Si bien el calendario de operación anual se ha cambiado, postergando en un mes la decisión (15 de diciembre) es difícil que se tengan resultados escolares definitivos en ese momento, por lo que debería existir una etapa de ajuste con los datos finales cuando estén disponible

La discusión de estas materias se insertan en un marco de dilemas que la política de fortalecimiento de la educación pública requiere enfrentar, que implica compatibilizar criterios técnicos de diseño de planes estratégicos de mediano plazo, con ajustes manuales, sustentados en diagnósticos sólidos, hitos sustantivos y variables e indiciadores que permitan un seguimiento adecuado y oportuno para la toma de decisiones. Ello implica además incorporar en grado importante a la comunidad escolar en este proceso y también que su seguimiento considere la gestión política local.

El segundo ámbito de dilemas, más propio de los SLE, es que el proceso de fortalecimiento de la educación pública, en el plano subnacional, implica, por una parte, una recentralización de la gestión. Esto tiene aparejado, ciertamente, dificultades no previstas a la fecha, que deberán resolverse gradualmente. Esto podrá realizarse con la ayuda de un Plan Estratégico adecuado, que además compatibilice las diversas culturas organizacionales de las unidades locales, las que han funcionado autónomamente por más de 40 años. En segundo lugar, deberá implementarse un proceso de fortalecimiento de lo público que vaya en contra de las corrientes dominantes que promueven su privatización directa o indirecta, elemento inédito en el proceso que se está implementando (Donoso et al., 2018).

Los debates que se han expuesto confirman la importancia de poder generar vínculos sustantivos entre la planificación local y el mejoramiento educativo, ratificando a Bryk et al. (2010), sobre la relevancia de generar las condiciones para que se instale una cultura en este plano y se alcancen impactos sustentables, esencialmente basados en una razonable independencia de la gestión local del marco político. En razón de ello, siguiendo a Anderson et al. (2012), se plantea lo relevante del fortalecimiento de la educación pública y la necesidad de fortalecer su institucionalidad en lo organizacional; para, de esta manera, impactar en el mejoramiento desde la política local. Confirmando lo planteado por Leithwood y Azah (2017), referente a que el sistema de educación pública local chileno tiene desafíos muy relevantes, que distan notablemente de los procesos de planificación estratégica local de aquellos territorios que han tenido experiencias exitosas en este plano

Adicionalmente, dada la crisis que se ha instalado en el sector público requiere de otros apoyos en materia de calificación y formación de directivos, disponibilidad y oportunidad de los recursos financieros, fomento de la participación de la comunidad y otras medidas que contribuyan en forma directa para que los sistemas locales de educación pública en Chile dispongan de un soporte sólido en este ámbito.

\section{Referencias}

Anderson, S., Mascall, B., Stiegelbauer, S., y Park, J. (2012). No one way: Differentiating school district leadership. Journal of Educational Change, 13:403-430.

Armijo, M. (2009). Manual de planificación estratégica e indicadores de desempeño en el sector público. ILPES/CEPAL.

Atria, F. (2014). Derechos sociales en educación: Un 
nuevo paradigma de lo público. LOM Editores, Santiago.

Ball, S. (1993). What is policy? texts, trajectories and toolboxes. Discourse, Studies in the Cultural Politics of Education, 13(2):10-17.

Ball, S. (2015). What is policy? 21 years later: reflections on the possibilities of policy research. Discourse: Studies in the Cultural Politics of Education.

Bellei, C. (2014). El estudio de la segregación socioeconómica y académica de la educación chilena. Estudios Pedagógicos, XXXIX(1):325-345.

Bellei, C. (2015). El Gran Experimento. Mercado y Privatización de la Educación Chilena. LOM Editores, Santiago de Chile.

Bellei, C., Cabalin, C., y Orellana, V. (2013). The 2011 chilean student movement against neoliberal educational policies. Studies in Education, $3(3)): 426-440$.

Bowe, R., Ball, S., y Gold, A. (1992). Reforming education and changing schools, case stu-dies in policy sociology. Routledge, London.

Bryk, A., Sebring, P., Allensworth, E., Lupescu, S., y Easton, J. (2010). Schools for Improvement. Lesson from chicago. The University of Chicago Press.

Consejo Asesor (2006). Consejo Asesor Presidencial para la Calidad de la Educación Informe Final. Presidencia de la República, Santiago de Chile.

Cox, C. (2003). Políticas Educacionales en el Cambio de Siglo. La reforma del sistema escolar en Chile. Editorial Universitaria, Santiago de Chile.

Donoso, S. y Benavides, N. (2017). Descentralización de la gestión de la educación pública e institucionalidad local en chile: El caso de los directores comunales de educación. Innovar, $27(64): 29-42$.

Donoso, S., Díaz, G., y Benavides, N. (2018). Propuesta de indicadores de gestión para la educación pública local chilena. Cadernos de Pesquisa.

Elacqua, G., Martínez, M., y Aninat, C. (2010). ¿cómo fortalecer la educación municipal? capacidad y responsabilidad política. En Martinic, S. y Elacqua, G., editores, ¿Fin del Ciclo? Cambio en la Gobernanza del Sistema Educativo, pp. 101-130. UNESCO - Pontificia Universidad Católica de Chile, Santiago de Chile.

Eyzaguirre, S. (2012). Fortalecimiento de la educación escolar pública: ¿desmunicipalización? Puntos de Referencia, 340.

García-Huidobro, J. (2010). Para hacer pública la educación pública. En Martinic, S. y. G. E., editor, ¿Fin de ciclo? Cambios en la gobernanza del sistema educativo ( $p$, pp. 81-100. UNESCO - PUC, Santiago de Chile.

Garretón, M. (2016). La ruptura entre política y sociedad. una introducción. En Garretón, M. A., editor, La gran Ruptura, Institucionalidad Política y Actores sociales en Chile del siglo XXI, pp. 11- 20. LOM ediciones, Santiago.

González, Á., González, M., y Galdames, S. (2015). El sostenedor como agente de cambio: el rol de los coordinadores técnicos en el apoyo a establecimientos municipales chilenos. Pensamiento Educativo, 52(1):47-64.

Jofré, G. (1988). Subvenciones en educación. Estudios Públicos, 32:31 -55.

Joiko, S. (2012). El cuasi-mercado educativo en chile: desarrollo y consecuencias. Diálogos Educativos, 23(12):148 -174.

Larrañaga, O., Peirano, C., y Falck, D. (2009). Una mirada al interior del sector municipal. En Marcel, M. y Raczynski, D., editores, La asignatura pendiente, pp. 77-97. Uqbar Editores y CIEPLAN, Santiago de Chile.

Leithwood, K. y Azah, V. (2017). Characteristics of high-performing school districts. Leadership and Policy in Schools, 16(1):27-53.

Mainardes, J. (2006). Abordagem do ciclo de políticas; uma contribucao para a análise de políticas educacionais. Revista Educação e Sociedade, 27:São Paulo.

Marcel, M. y Raczynski, D. (2009). La Asignatura Pendiente. Claves para la revalidación de la educación pública de gestión local en Chile. Uqbar editores, Santiago de Chile.

Mazuccato, M. (2014). El Estado Emprendedor. RBE Libros, Madrid.

MINEDUC (2005). Marco para la Buena Dirección. Ministerio de Educación, Santiago de Chile.

MINEDUC (2009). Guía PADEM. 
MINEDUC (2016). Guión Metodológico Comité PA$D E M$ 1.0. Ministerio de Educación, Santiago de Chile.

MINEDUC (2017). Guión Metodológico Comité PA$D E M$ 3.0. Ministerio de Educación, Santiago de Chile.

MINEDUC (2018). Estadísticas de matricula. Centro de estudios del Ministerio de Educación, Santiago de Chile.

Núñez, I. y Weinstein, J. (2010). Chile: Una reforma educacional ¿sin reforma del ministerio? (1990 -2007). En Aguerrondo, I., editor, Institucionalidad de los Ministerio de Educación. Los procesos de reforma educativa de Argentina y Chile en los años 90, pp. 95-184. UNESCOIIEP, París.

OCDE (2004). Chile. Revisión de las políticas $\mathrm{Na-}$ cionales de Educación. Organización para la Cooperación y el Desarrollo, París.

OCDE (2017). Educación en Chile. Evaluación de Políticas nacionales de Educación. Organización para la Cooperación y el Desarrollo, París.

Oyarzo, P. (2012). Procesos de gestión del departamento comunal de educación municipal de talca: Análisis y propuestas de mejoramiento.

PNUD (2017). Desiguales. Orígenes, cambios y desafios de la brecha Social en Chile. Programa de las Naciones Unidas para el Desarrollo, Santiago de Chile.

Politeia (2008). Estudio mejoramiento de la gestión y la calidad de la gestión municipal. Informe ejecutivo por encargo de DIPLAP. MINEDUC, Santiago.

Raczynski, D. y Salinas, D. (2009). Prioridades, actores y procesos en la gestión municipal de la educación. En Marcel, M. y Raczynski, D., editores, La asignatura pendiente Claves pa-ra la Revalidación de la Educación Pública de Gestión Local en Chile, pp. 135 -176. Uqbar Editores, Santiago de Chile.

Ruiz, C. (2016). Crisis política en chile: neoliberalismo, cambios sociales y democracia. En Garretón, M., editor, La gran Ruptura, Institucionalidad Politica y Actores socia-les en Chile del siglo XXI, pp. $83-108$. LOM ediciones, Santiago.
Saavedra, P. (2009). El desarrollo del padem en la provincia de curicó.

SINIM (2017). Sistema Nacional de información Municipal. Santiago de Chile.

Treviño, E., Valenzuela, J., y Villalobos, C. (2018). Agrupamiento por habilidad académica en el sistema escolar nueva evidencia para comprender las desigualdades del sistema educativo chileno. Revista Mexicana de Investigación Educativa, 23(76):45-71.

Valenzuela, J., Bellei, C., y de los Ríos, D. (2014). Socio-economic school segregation in a market oriented educational system. the case of chile. Journal of Education Policy, 29(2):217-241.

Waissbluth, M. (2013). Cambio de Rumbo, Una nueva vía chilena a la educación. Ramdom House Mondadori S.A, Santiago de Chile.

Zamorano, L. (2006). Descentralización de la educación y el desempeño de los municipios. Instituto Chileno de Estudios Municipales. ICHEM, Santiago de Chile. 